\title{
Breakpoint Localization Using Array-CGH in Three Siblings With an Unbalanced 4q;16q Translocation and Childhood Apraxia of Speech (CAS)
}

\author{
Lawrence D. Shriberg, ${ }^{1}{ }^{*}$ Kathy J. Jakielski, ${ }^{2}$ and Hatem El-Shanti ${ }^{3}$ \\ ${ }^{1}$ Waisman Center, University of Wisconsin-Madison, Madison, Wisconsin \\ ${ }^{2}$ Department of Communication Sciences and Disorders, Augustana College, Rock Island, Illinois \\ ${ }^{3}$ Department of Pediatrics, University of Iowa, Iowa City, Iowa
}

Received 25 September 2007; Accepted 24 March 2008

\begin{abstract}
We report clinical, cytogenetic, and comparative genomic hybridization findings for three siblings with an unbalanced 4q;16q translocation, minor malformations, and cognitive abnormalities, including childhood apraxia of speech, a rare, severe motor speech disorder. Breakpoint findings indicate that in addition to possible contributions from duplicated genes on chromosome 16, haploinsufficiency of one or more of 11 genes deleted in the telomeric region of the long arm of chromosome 4 is the likely cause of the speech disorder, the associated impairments in cognition and language, and the dysmorphic features. The present findings are the first to
\end{abstract}

document childhood apraxia of speech in a multiplex family using contemporary speech measures. We suggest that genotype-phenotype studies of childhood apraxia of speech occurring in complex neurodevelopmental disorders can elucidate the pathophysiology of this disorder. (c) 2008 Wiley-Liss, Inc.

Key words: articulatory apraxia; chromosome translocation; language disorder; speech disorder; FOXP2

\section{How to cite this article: Shriberg LD, Jakielski KJ, El-Shanti H. 2008. Breakpoint localization using array-CGH in three siblings with an unbalanced 4q;16q translocation and childhood apraxia of speech (CAS). Am J Med Genet Part A 146A:2227-2233.}

\section{INTRODUCTION}

Childhood apraxia of speech (CAS) is a rare motor speech disorder that places a child at risk for significant and persistent difficulties in speech and language, with consequences for challenges in educational, social, and vocational domains. As a secondary sign in the context of complex neurodevelopmental disorders, CAS is frequently comorbid with cognitive impairment, language impairment, nonverbal oral apraxia, and/or dysmorphic features. The American Speech-LanguageHearing Association recently endorsed CAS as the classificatory term for a childhood disorder of speech praxis, replacing terms such as Developmental Verbal Dyspraxia (DVD) and Developmental Apraxia of Speech (DAS) [ASHA, 2007]. The ASHA technical report defines CAS as:

...a neurological childhood (pediatric) speech sound disorder in which the precision and consistency of movements underlying speech are impaired in the absence of neuromuscular deficits (e.g., abnormal reflexes, abnormal tone). ...The core impairment in planning and/or programming spatiotemporal parameters of movement sequences results in errors in speech sound production and prosody. (p. 3)

Genetic studies of CAS have been catalyzed by the widely cited research series on the London family in which a mutation in FOXP2, nonverbal oral apraxia, and a severe speech sound disorder consistent with apraxia of speech was identified in half of the members of a then three-generation family [Lai et al., 2000, 2001], reviewed in [Fisher, 2007]. A problem with this study series and others in idiopathic CAS is that the inclusionary signs used to classify family members as positive for CAS were obtained from non-standardized speech assessment protocols. We

*Correspondence to: Lawrence D. Shriberg, Room 439, Waisman Center University of Wisconsin-Madison 1500 Highland Avenue Madison, WI 53705. E-mail: shriberg@waisman.wisc.edu

DOI 10.1002/ajmg.a.32363 
have proposed that programmatic research in CAS requires a standardized protocol of perceptual and instrumental measures that quantify relevant features of apraxia of speech as it presents in four contexts: in adult acquired apraxia of speech (AOS), as a consequence of neurological disorder in children (e.g., infection, trauma), as a secondary sign in complex neurodevelopmental disorders, and as an idiopathic speech sound disorder [Shriberg, in press]. There is considerable public health interest in the latter putative subtype because its reported prevalence has risen markedly in the past approximately two decades, but studies indicate high rates of false positives [Davis et al., 1998; Campbell, 2003; ASHA, 2007]. Here we describe the results of a welldeveloped speech assessment protocol to document CAS in a multiplex family in which each of three siblings has an unbalanced 4q;16q translocation (monosomy $4 \mathrm{q}$ and trisomy $16 \mathrm{q}$ ) inherited from their father who has a balanced 4q;16q translocation. The goal was to identify the breakpoints on each chromosome, with the possibility of identifying novel candidate genes and loci that may play a role in CAS.

\section{MATERIALS AND METHODS}

\section{Speech Analyses}

The three patients (at ages 16 years 9 months, 12 years 3 months, and 10 years 11 months) and their father completed a 45-min speech, prosody, and voice assessment (administered by KJJ). This research project was approved by the University of Wisconsin-Madison Institutional Review Board following federal regulations under 45CFR46. The protocol included a conversational speech sample, the Goldman-Fristoe Test of Articulation 2 [Goldman and Fristoe, 2000], and a series of brief tasks requiring spontaneous and imitative speech responses at the word, phrase, and sentence level. As described in detail in Shriberg et al. [2006], the protocol was designed to discriminate CAS from three other possible clinical classifications: typical speech, speech delay, or dysartbria. The digital audio recordings were processed in the PEPPER software platform [Shriberg et al., 2001] at the University of Wisconsin-Madison, using methods for transcription, prosody-voice coding, and acoustic analysis of typical and disordered speech production.

\section{Genetic Analyses}

The patients were evaluated at the University of Iowa Medical Genetics outpatient clinic and their clinical records were reviewed by one of the authors (HES) after informed consent. All family members were genotyped with microsatellite markers distributed along the terminal parts of the long arms of chromosomes 4 and 16. The breakpoint on chromosome 4 was mapped by this method to a $400 \mathrm{~kb}$ region, while the breakpoint on chromosome 16 could not be mapped due to the restrictions imposed by the microsatellite marker genotyping approach.

We subsequently employed custom microarray comparative genomic hybridization (CGH) to determine the breakpoints on chromosomes 4 and 16 by calculating the copy number in the regions of interest. An array CGH of approximately 71,000 probes on telomeric end of the long arm of chromosome 4, spaced at a median interval of 50 base pairs (Roche NimbleGen Systems Inc.), was custom designed. Because the breakpoint on chromosome 16 had not been determined, we elected to cover the whole chromosome, but with lower resolution than 4q. Also included were approximately 81,000 probes for a region on chromosome 7 that contains FOXP2. DNA was isolated from peripheral blood using standard protocols from the three siblings and a control. The scanned output was extracted according to signal intensity of each probe, coordinated with the genomic information, and the data normalized. The output file provided the predicted copy number of each probe. All family members had the custom microarray CGH performed. Despite the high resolution of the array CGH, to confirm the breakpoint on chromosome 4, we genotyped the whole family for selected SNPs that cross the breakpoint by direct DNA sequencing. We did not perform any confirmatory testing for the breakpoint on chromosome 16.

\section{CASE REPORTS}

The three patients reported here (Patients $1-3$ ) are siblings referred to one of the authors (LDS) as candidates for a study to examine the genetic bases of CAS. Each of the children has been treated for a severe speech sound disorder consistent with CAS since early childhood. Due to the presence of congenital anomalies and dysmorphic features, the three children had been evaluated by karyotype and sub-telomere FISH testing and had been seen by a clinical geneticist. The father was judged as unaffected and the family history is otherwise negative for speech disorders.

Prior to our reassessment, the most recent comprehensive cognitive, language, and speech assessments using standardized tests had been completed when Patients 1-3, respectively, were 13 years, 8 years 7 months, and 7 years 2 months. Results on the Comprehensive Test of Nonverbal Intelligence [Hammill et al., 1996] indicated that they had cognitive deficits ranging from 1.5 standard deviations below the mean for their age (Patients 2 and 3 ) to 3.0 standard deviations below the mean (Patient 1). All three children had similar and severe receptive and expressive language disorders characterized by deficits in semantic, morphosyntactic, syntactic, 
discourse, narrative, and literacy domains. The speech profiles for the siblings were also similar, characterized by poor imitative abilities, incomplete sound inventories, multiple vowel errors, inconsistent word productions, increasing number of errors as word length increased, unintelligible conversational speech, slow and dysrhythmic diadochokinetic rates, and fluctuating nasal resonance. These characteristics are consistent with, but not specific for, a diagnosis of CAS, the disorder for which they had each been receiving treatment.

Patient 1 was 15 years old at her genetics evaluation. Her medical records indicated repeated otitis media, otitis media with effusion, and several pressure equalization tube placements. There were fluctuating conductive hearing loss and occular problems in the form of different size globes and amblyopia. She had a history of seizures with abnormal left hemisphere EEG and scoliosis. Her height was $153.6 \mathrm{~cm}$ ( 10 th centile), her weight was $48 \mathrm{~kg}$ ( $\sim 25$ th centile), and her OFC was $53.5 \mathrm{~cm}$ ( $\sim 1$ SD below the mean). She had frontal bossing with right frontal upsweep, epicanthal folds, and discrepant eye globe size (Fig. 1). She had low set and posteriorly rotated ears, hypoplastic alae nasi, and bulbous nasal tip. The uvula was absent with indication of velopharyngeal insufficiency (nasal tone). The lower and upper central incisors were widely separated, the upper lateral incisors were pegged, and the canines were small. There was prominent discoloration and translucency of all teeth. The hands showed thenar and hypothenar hypoplasia, bilateral fourth and fifth finger clinodactyly, and abnormally placed thumbs.
Patient 2 was 10 years 6 months old at the time of her evaluation. Her medical history indicated repeated otitis media, otitis media with effusion, several pressure equalization tube placements, fluctuating conductive hearing loss, astigmatism, and amblyopia. There was a history of scoliosis, vesico-ureteric reflux, and congenital hip dysplasia. Her height was $136 \mathrm{~cm}(\sim 25$ th centile), her weight was $39 \mathrm{~kg}$ ( $\sim 75$ th centile), and her OFC was $54.5 \mathrm{~cm}$ ( $\sim 1.5$ SD above the mean). She had frontal bossing and posteriorly rotated ears with ear pits (Fig. 1). The lower teeth were crowded with hypodontia of the upper teeth. There was prominent discoloration and translucency of all teeth. The hands showed bilateral fifth finger clinodactyly and the right thumb was smaller than the left. She had bilateral partial cutaneous syndactyly of the first and second toe (separated at the nails only) (Fig. 2).

Patient 3 was 9 years old at the time of his evaluation. His medical history indicated repeated otitis media, otitis media with effusion, several pressure equalization tube placements and conductive hearing loss. There was a history of pulmonary hypertension and patent ductus arteriosis at birth. His height was $130 \mathrm{~cm}(\sim 25$ th centile), his weight was $33 \mathrm{~kg}$ ( $\sim 75$ th centile), and his OFC was $53.5 \mathrm{~cm}$ ( $\sim$ at the mean). He had frontal bossing and a hypopigmented spot on the left temporal area with uneven borders (Fig. 1). He had epicanthal folds and posteriorly rotated ears, hypoplastic alae nasi, and bulbous nasal tip. There was hypodontia of the upper teeth. There was prominent discoloration and translucency of all teeth. The hands showed bilateral fifth finger clinodactyly.

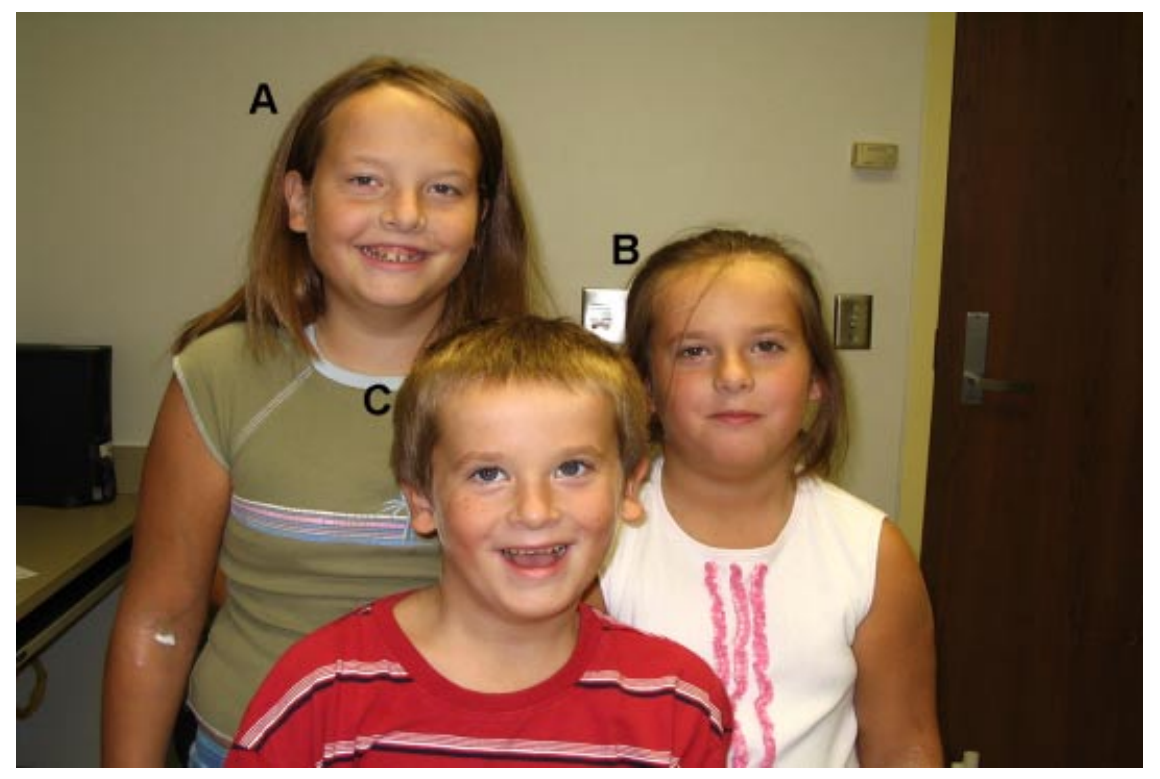

FIG. 1. Photograph of the three siblings, arranged vertically from $\mathbf{A}$ oldest to $\mathbf{C}$ youngest, showing common dysmorphic features, including frontal bossing, epicanthal folds, posterior ear rotation, hypoplastic alae nasi, and crowded, unusual, or decreased number of teeth. [Color figure can be viewed in the online issue, which is available at www.interscience.wiley.com.] 


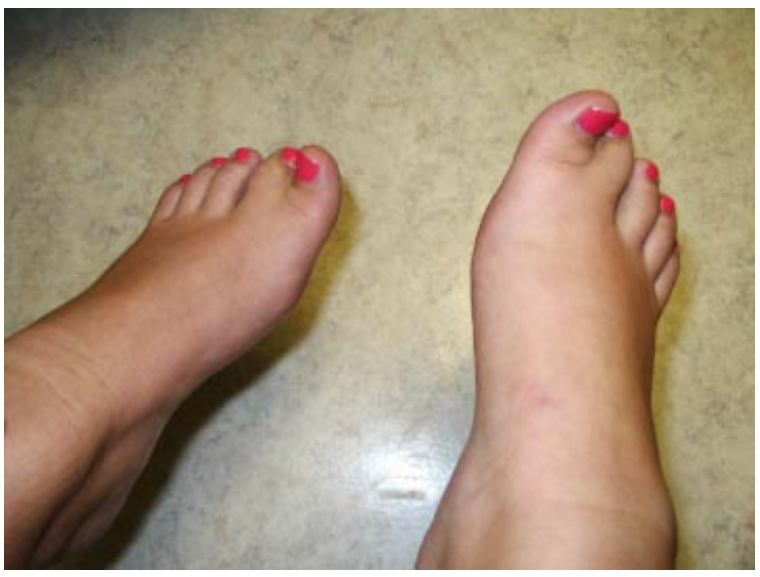

FIg. 2. Photograph of feet of Patient 2. [Color figure can be viewed in the online issue, which is available at www.interscience.wiley.com.]

The speech data are presented here in aggregate to avoid duplication. The father's speech data are not included because his case history data and our assessment indicated that he had typical speech. Each of the three patients had significantly lower scores for speech precision than children with typical speech (more than 1 SD below the mean) on these measures (Table I). They also had lower scores than adolescents with persistent speech delay, using data from such children in our database.

Additional measures have been developed to provide reliable diagnosis of CAS [Shriberg et al., 1997, 1999, 2003a,b; Odell and Shriberg, 2001]. Comparison adolescent data were available only for the children with typical speech just described. All three patients had significantly lower performance than the typical speakers on measures 4 and 5 and the composite, which measures error type and consistency. For measures 6-8, which provide acoustic information on timing and stress, Patient 2's scores were not significantly different on any measure, Patient 1 had a significantly different score on one measure, and Patient 3 had a significantly different score on all three of the measures. Thus, the three patients had lower scores on three to five of the measures proposed to be diagnostic for CAS. Importantly, the type and pattern of their errors is specific for CAS, rather than for severe speech delay or dysarthria. The relative magnitudes of scores and the two composites indicate that Patients 1 and 3, the oldest and youngest of the siblings, respectively, had the most severe and persistent CAS phenotype.

\section{Genetic Findings}

Results of fluorescence in situ hybridization (FISH) testing to detect subtelomeric rearrangements revealed an unbalanced telomeric translocation $\operatorname{der}(4) \mathrm{t}(4 ; 16)$ (qter-,qter+) involving the subtelomeric sequences in all three siblings (Fig. 3). A karyotype and FISH analysis of the father demonstrated a balanced translocation $\mathrm{t}(4 \mathrm{q} ; 16 \mathrm{q})$ (qter-, qter+;qter-,qter+). The karyotype and FISH analysis of the mother showed no chromosomal abnormality.

The array-CGH analyses indicated that each patient has the same breakpoint on chromosomes 4 and 16 . As indicated for one of the siblings in Figure 4 , the breakpoint on chromosome 4 is at $187.8 \mathrm{Mb}$ (band 4q35.2), within the genomic structure of FAT. The breakpoint on chromosome 16 is at $78.6 \mathrm{Mb}$ (band 16q23.2) and does not disrupt any genes. The deleted region on chromosome 4 creates haploinsufficiency for the genes in the telomeric region $(3.46 \mathrm{Mb})$ whereas the duplicated region on chromosome 16 produces trisomy for the telomeric region (10.2 Mb).

The genes that are deleted on chromosome 4 are listed in Table II. The duplicated region on

TABLE I. Descriptive and Diagnostic Speech Findings for the Three Siblings

\begin{tabular}{|c|c|c|c|c|c|c|c|}
\hline \multirow[b]{3}{*}{ Domain } & \multicolumn{4}{|c|}{ Measure } & \multicolumn{3}{|c|}{ Scores $^{\mathrm{a}}$} \\
\hline & \multirow[b]{2}{*}{ Title } & \multicolumn{3}{|c|}{ Type } & \multirow[b]{2}{*}{ P1 } & \multirow[b]{2}{*}{ P2 } & \multirow[b]{2}{*}{ P3 } \\
\hline & & Perceptual & Acoustic & Diagnostic for CAS & & & \\
\hline \multirow[t]{4}{*}{ Precision } & 1. Percentage of consonants correct revised & $\mathrm{X}$ & & & $82.6 \% \%^{* \dagger}$ & $86.5 \%{ }^{* \dagger}$ & $78.7 \% \%^{* \dagger}$ \\
\hline & 2. Percentage of phonemic diphthongs correct & $\mathrm{X}$ & & & $84.4 \% \%^{* \dagger}$ & $97.2 \%^{* \dagger}$ & $80.5 \% \%^{* \dagger}$ \\
\hline & 3. Intelligibility index & $\mathrm{X}$ & & & $77.0 \%{ }^{* \dagger}$ & $82.4 \%^{* \dagger}$ & $70.8 \% \%^{* \dagger}$ \\
\hline &  & & & & $81.3 \%^{* \dagger}$ & $88.7 \% \%^{* \dagger}$ & $76.7 \% \%^{* \dagger}$ \\
\hline \multirow{3}{*}{$\begin{array}{l}\text { Error type and } \\
\text { consistency }\end{array}$} & 4. Error type index & $\mathrm{X}$ & & $\mathrm{X}$ & $63.2 \%^{*}$ & $72.2 \% *$ & $77.3 \% *$ \\
\hline & 5. Error consistency index & $\mathrm{X}$ & & $\mathrm{X}$ & $39.3 \%{ }^{*}$ & $54.5 \% *$ & $49.3 \%{ }^{*}$ \\
\hline & Error type and consistency composite $(\overline{\mathrm{X}}: 1-3)$ & & & & $51.3 \% *$ & $63.4 \% \%^{*}$ & $60.8 \% *$ \\
\hline \multirow[t]{3}{*}{ Timing and stress } & 6. Pairwise variability index & & $\mathrm{X}$ & $\mathrm{X}$ & 57.1 & 49.0 & $50.5^{*}$ \\
\hline & 7. Speech-pause variability index & & $\mathrm{X}$ & $\mathrm{X}$ & $1.33^{*}$ & 1.30 & $1.60^{*}$ \\
\hline & 8. Lexical stress index & & $X$ & $\mathrm{X}$ & 1.29 & 1.31 & $1.70^{*}$ \\
\hline
\end{tabular}

${ }^{a}$ High scores indicate better performance for measures 1-6 and poorer performance for measures 7 and 8.

*Significantly poorer scores (by at least one standard deviation) than typical speakers within one year of age of each patient.

${ }^{\dagger}$ Significantly poorer scores than adolescent speakers with persistent speech delay. Comparative data for these speakers are currently not available for measures $4-8$. 


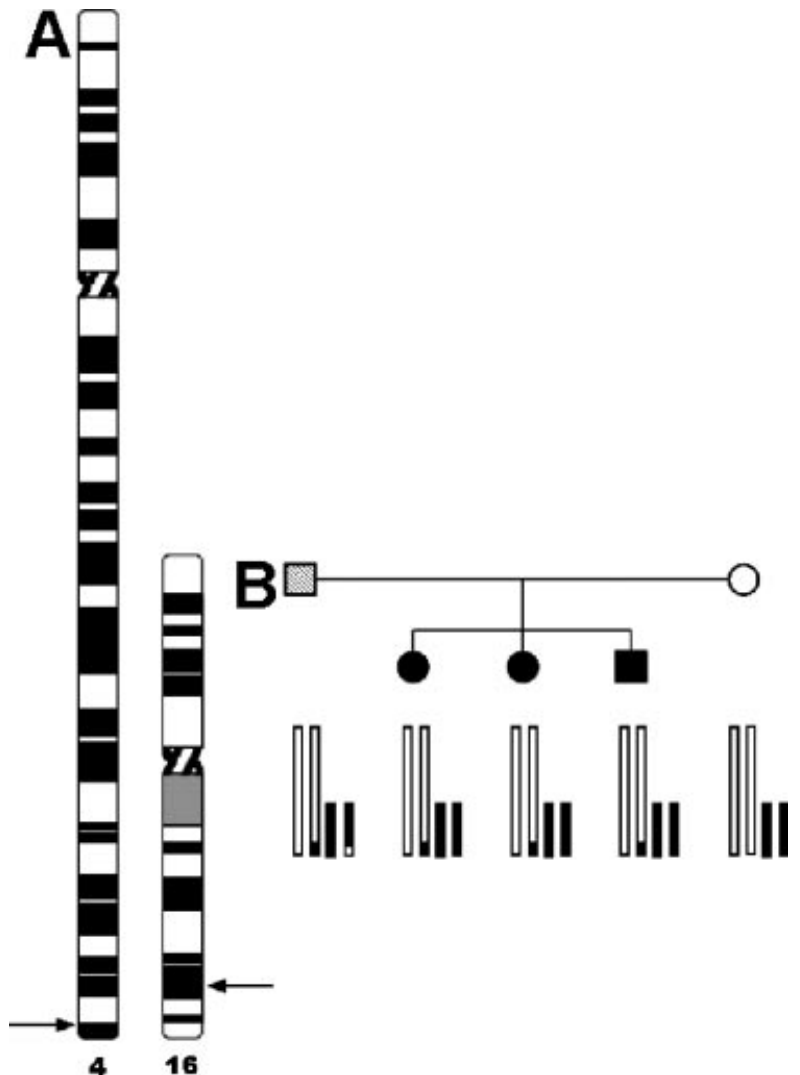

FIG. 3. A: Pedigree of the family with $\mathbf{B}$ diagrammatic representation of the chromosome rearrangement present in each family member. The father carries the balanced form of the translocation and does not have any copy number changes. The three children are monosomic for $4 \mathrm{q}$ and trisomic for $16 \mathrm{q}$

chromosome 16 contains more than 100 known and predicted genes. Findings for the probes in the chromosome seven region that contains FOXP2 did not indicate any copy number abnormalities. Neither the father nor the mother had any deletions or duplications in the three examined regions by array $\mathrm{CGH}$, which indicates that the father has a balanced translocation. All genotyped SNPs telomeric to the breakpoint on chromosome 4 were hemizygous in the three patients, confirming the break point.

\section{DISCUSSION}

The father of the three children carries a balanced translocation producing unbalanced offspring through adjacent I segregation of chromosomes at meiosis. However, it is surprising that the father did not produce any chromosomally balanced offspring, despite the fact that alternate segregation is the most likely mode expected for this reciprocal translocation, which would produce balanced gametes.

The presence of dysmorphic features and congenital anomalies in each patient is likely explained by their unbalanced chromosomal complement, including both the haploinsufficiency in the deleted region of chromosome 4 and possibly gene dosage imbalances associated with the extra chromosomal material of chromosome 16. All of the children share common facial and dental features, as well as hand and foot malformations. A small number of trisomy 16q cases have been described [Brisset et al., 2002]. Most of the reported cases, similar to those reported here, are due to malsegregation of a balanced parental translocation. The three siblings reported here have several findings common in partial trisomy 16q, such as clinodactyly, prominent forehead or frontal bossing, epicanthal folds, low set posteriorly rotated ears, and vesico-ureteric reflux.

Although the three patients are deleted for the FRG1 gene, they do not manifest the facioscaplohumeral muscular dystrophy (FSHD) phenotype because it is caused by repeat mediated upregulation of gene expression [Tupler and Gabellini, 2004; Gabellini et al., 2006]. We conclude that the hearing loss in the three children and the epilepsy in one of them is probably not related to the FSHD deletion.


FIG. 4. Microarray CGH from one of the three children showing the breakpoint on chromosome 4 at 187.8 Mb (band $4 \mathrm{q} 35.2$ ) and the breakpoint on chromosome 16 at $78.6 \mathrm{Mb}$ (band 16q23.2). [Color figure can be viewed in the online issue, which is available at www.interscience.wiley.com.] 
TABLE II. Genes Deleted from the Telomeric Region of the Long Arm of Chromosome 4

\begin{tabular}{rcl}
\hline & Gene symbol & \multicolumn{1}{c}{ Description } \\
\hline 1 & FAT (partial) & Tumor suppressor gene with cadherin and EGF domains \\
2 & ZFP42 & Zinc-finger protein with zinc-finger $\mathrm{C}_{2} \mathrm{H}_{2}$ type domain \\
3 & FLJ25801 & Unknown function, no ortholog in other species \\
4 & TRIML1 & Tripartite motif family like 1 \\
5 & FLJ38649 & Unknown function, no ortholog in other species \\
6 & $B C O 87857$ & Unknown function, no ortholog in other species \\
7 & $F R G 1$ & Deleted in fascioscapulohumeral muscular dystrophy (FSH) \\
8 & TUBB4Q & Tubulin, $\beta$ polypeptide 4, member Q \\
9 & $D U X 4 C$ & Double homeobox 4C \\
10 & $F R G 2$ & Related to FSH \\
11 & $D U X 4$ & Double homeobox 4 \\
\hline
\end{tabular}

Of the 11 deleted genes on chromosome 4 , threeFLJ25801, FLJ38649, BC087857-are without known functions and have no ortholog in other species. These genes are of particular importance for further study since speech is unique to humans. Also, two genes, DUX4C and CUX4, have homeobox developmental protein functions similar to the functions of the FOX family of genes. The roles of these genes in speech development should be explored.

The trisomic region on chromosome 16 in the three children contains over 100 predicted or known genes. The involvement of the chromosome 16 q24 region in specific language impairment has replicated in two genome-wide association studies [SLI Consortium, 2002, 2004] and in a study of these data using multivariant variancecomponents technique [Monaco, 2007]. Of special interest among the genes in this region are FOXF1, FOXC2, and FOXL1, which are forkhead transcription factors.

The present findings illustrate the potential contributions of genetic studies to eventual explanatory accounts of CAS as it occurs idiopathically and in complex neurodevelopmental disorders. The contributions of the genes noted above and others to both the similarities and heterogeneities observed in the physical and speech findings should be explored in other children with CAS, including those with genetic deficits affecting FOXP2. As concluded in a prior report of two family members with CAS associated with a 7;13 translocation affecting FOXP2 [Shriberg et al., 2006], lack of published information on affected KE family members' CAS phenotypes precludes direct comparison of speech findings with the present data. Similarly, the lack of detailed speech data in 18 reports of suspected CAS in the context of genetic disorders [Shriberg, in press] prohibits potentially informative genotype-phenotype comparison with the present data. We suggest that use of a standardized instrumental protocol sensitive to, and specific for, the CAS phenotype is critical for an eventual understanding of the genetic substrates and pathophysiology of this challenging pediatric speech sound disorder.

\section{ACKNOWLEDGMENTS}

This work was supported by a grant from the National Institute on Deafness and Other Communicative Disorders (DC00496), a Core Grant to the Waisman Center (HD03352) from the National Institute of Child Health and Development, and a support grant from the Board of Visitors, Waisman Center. The authors thank the patients and their parents for their support throughout this project. We also thank our laboratory colleagues at the University of Wisconsin, Augustana College, and the University of Iowa, and consultants at Roche NimbleGen Systems Inc. for their contributions to this report.

\section{REFERENCES}

American Speech-Language-Hearing Association. 2007. Childhood apraxia of speech [Technical Report]. Available from www.asha.org/policy.

Brisset S, Joly G, Ozilou C, Lapierre JM, Gosset P, LeLorc'h M, Raoul O, Turleau C, Vekemans M, Romana SP. 2002. Molecular characterization of partial trisomy 16q24.1-qter: Clinical report and review of the literature. Am J Med Genet 113: 339-345.

Campbell TF. 2003. Childhood apraxia of speech: Clinical symptoms and speech characteristics. In: Shriberg LD, Campbell TF, editors. Proceedings of the 2002 childhood apraxia of speech research symposium, The Hendrix Foundation, Carlsbad. p 37-40.

Davis B, Jakielski K, Marquardt T. 1998. Developmental apraxia of speech: Determiners of differential diagnosis. Clin Linguist Phon 12:25-45.

Fisher SE. 2007. Molecular windows into speech and language disorders. Folia Phoniatr Logop 59:130-140.

Gabellini D, D'Antona G, Moggio M, Prelle A, Zecca C, Adami R, Angeletti B, Ciscato P, Pellegrino MA, Bottinelli R, Green MR, Tupler R. 2006. Facioscapulohumeral muscular dystrophy in mice overexpressing FRG1. Nature 439:973-977.

Goldman R, Fristoe M. 2000. Goldman-Fristoe test of articulation. 2nd ed. Circle Pines, MN: American Guidance Service, Inc.

Hammill DD, Pearson NA, Wiederholt JL. 1996. Comprehensive test of nonverbal intelligence. Austin, TX: Pro-Ed.

Lai CSL, Fisher SE, Hurst JA, Levy ER, Hodgson S, Fox M, Jeremiah $S$, Povey S, Jamison DC, Green ED, Vargha-Khadem F, Monaco AP. 2000. The SPCH1 region on human 7q31: Genomic characterization of the critical interval and localization of translocations associated with speech and language disorder. Am J Hum Genet 67:357-368. 
Lai CSL, Fisher SE, Hurst JA, Vargha-Khadem F, Monaco AP. 2001. A forkhead-domain gene is mutated in a severe speech and language disorder. Nature 413:519-523.

Monaco AP. 2007. Multivariate linkage analysis of specific language impairment (SLI). Ann Hum Genet 71:660-673.

Odell KH, Shriberg LD. 2001. Prosody-voice characteristics of children and adults with apraxia of speech. Clin Linguist Phon 15:275-307.

Shriberg LD. In press: A. neurodevelopmental framework for research in childhood apraxia of speech. In: Maassen B, van Lieshout $\mathrm{P}$, editors. Speech motor control: New developments in basic and applied research. United Kingdom: Oxford University Press.

Shriberg LD, Allen CT, McSweeny JL, Wilson DL. 2001. PEPPER: Programs to examine phonetic and phonologic evaluation records [Computer software]. Madison, WI: Waisman Center Research Computing Facility, University of WisconsinMadison.

Shriberg LD, Aram DM, Kwiatkowski J. 1997. Developmental apraxia of speech. I. Descriptive and theoretical perspectives. J Speech Lang Hear Res 40:273-285.

Shriberg LD, Ballard KJ, Tomblin JB, Duffy JR, Odell KH, Williams CA. 2006. Speech, prosody, and voice characteristics of a mother and daughter with a 7;13 translocation affecting FOXP2. J Speech Lang Hear Res 49:500-525.

Shriberg LD, Campbell TF, Karlsson HB, Brown RL, McSweeny JL, Nadler CJ. 2003a. A diagnostic marker for childhood apraxia of speech: The lexical stress ratio. Clin Linguist Phon 17:549574 .

Shriberg LD, Green JR, Campbell TF, McSweeny JL, Scheer A. 2003b. A diagnostic marker for childhood apraxia of speech: The coefficient of variation ratio. Clin Linguist Phon 17:575595.

Shriberg LD, Tomblin JB, McSweeny JL. 1999. Prevalence of speech delay in 6-year-old children and comorbidity with language impairment. J Speech Lang Hear Res 42:14611481.

SLI Consortium. 2002. A genomewide scan identifies two novel loci involved in specific language impairment. Am J Hum Genet 70:384-398.

SLI Consortium. 2004. Highly significant linkage to the SLI1 locus in an expanded sample of individuals affected by specific language impairment. Am J Hum Genet 74:1225-1238.

Tupler R, Gabellini D. 2004. Molecular basis of facioscapulohumeral muscular dystrophy. Cell Mol Life Sci 61:557566. 